University of Nebraska - Lincoln

DigitalCommons@University of Nebraska - Lincoln

6-1995

\title{
Accessing Earth System Science Data and Applications Through High-Bandwidth Networks
}

\author{
R. Vetter \\ IEE
}

M. Ali

University of North Dakota

M. Daily

IEE

J. Gabrynowic

University of North Dakota, Grand Forks, $N$

Sunil G. Narumalani

University of Nebraska-Lincoln, snarumalani1@unl.edu

See next page for additional authors

Follow this and additional works at: https://digitalcommons.unl.edu/csearticles

Part of the Computer Sciences Commons

Vetter, R.; Ali, M.; Daily, M.; Gabrynowic, J.; Narumalani, Sunil G.; Nygard, K.; Perrizo, W.; Ram, P.;

Reichenbach, S.; Seielstad, G. A.; and White, W., "Accessing Earth System Science Data and Applications Through High-Bandwidth Networks" (1995). CSE Journal Articles. 7.

https://digitalcommons.unl.edu/csearticles/7

This Article is brought to you for free and open access by the Computer Science and Engineering, Department of at DigitalCommons@University of Nebraska - Lincoln. It has been accepted for inclusion in CSE Journal Articles by an authorized administrator of DigitalCommons@University of Nebraska - Lincoln. 


\section{Authors}

R. Vetter, M. Ali, M. Daily, J. Gabrynowic, Sunil G. Narumalani, K. Nygard, W. Perrizo, P. Ram, S.

Reichenbach, G. A. Seielstad, and W. White

This article is available at DigitalCommons@University of Nebraska - Lincoln: https://digitalcommons.unl.edu/ 


\title{
Accessing Earth System Science Data and Applications Through High-Bandwidth Networks
}

\author{
R. Vetter, Member, IEEE, M. Ali, M. Daily, Member, IEEE, J. Gabrynowicz, S. Narumalani, \\ K. Nygard, Member, IEEE, W. Perrizo, Member, IEEE, P. Ram, Member, IEEE, \\ S. Reichenbach, Member, IEEE, G. A. Seielstad, and W. White, Member, IEEE
}

\begin{abstract}
In this paper, we discuss gigabit network applications enabled by "Mission to Planet Earth," an international effort to monitor the Earth as a system. We describe the design of a network architecture to support applications developed as part of this program; introduce a new component, public access resource centers (PARC's); and discuss how PARC's would facilitate access by users outside the traditional research community. We also describe how a particular class of users, agriculture users, might benefit from access to data collected as part of the Mission to Planet Earth program and delivered in a value-added form to them by a so-called AgPARC. The suggested architecture requires the deployment of high-bandwidth networks.
\end{abstract}

\section{INTRODUCTION}

$\mathbf{T}$ HE Global Change Research Program is a long-term international effort seeking to develop a better understanding of the Earth as a system and how that system might be changing. The program is designed to assess the Earth's ecological condition, understand its processes and how they interact, predict its future state, and find ways to ensure that the collective actions of humanity in the global environment do not undermine the Earth's viability as a system [9]. In the United States, several government agencies are contributing to the global change research program. One of the major contributors is the National Aeronautics and Space Administration (NASA), which has initiated a "Mission to Planet Earth" (MTPE) Program that uses space-and groundbased measurement systems to provide sustained observations of the Earth over a fifteen year period and to monitor trends in the various variables measured. The Earth Observing System (EOS) is the centerpiece of NASA's MTPE program [9]. The EOS consists of a series of polar-orbiting and low-inclination satellites, each satellite bearing several sensors, for long-term

Manuscript received August 1, 1994; revised January 17, 1995. This work was supported in part by Contract ECS-00011, Hughes Applied Information Systems.

R. Vetter and W. Perrizo are with the Department of Computer Science and Operations Research, North Dakota State University, Fargo, ND 58105 USA.

M. Ali, P. Ram, and W. White are with the Department of Computer Science, University of North Dakota, Grand Forks, ND 58202 USA.

M. Daily is with Hughes Research Laboratories, Malibu, CA 90265 USA

J. Gabrynowicz is with the Department of Space Studies, University of

North Dakota, Grand Forks, ND 58202 USA.

$\mathrm{S}$. Narumalani is with the Department of Geography, University of $\mathrm{Ne}$ braska, Lincoln, NB 68588 USA.

K. Nygard and G. Seielstad are with the Center for Aerospace Sciences,

University of North Dakota, Grand Forks, ND 58202 USA

$S$. Reichenbach is with the Computer Science and Engineering Department

University of Nebraska, Lincoln, NB 68588 USA.

IEEE Log Number 9410351. global observations of the land surface, biosphere, solid Earth, atmosphere, polar ice, and oceans. The Earth Observing System Data and Information System (EOSDIS) will manage data resulting from NASA's Earth science research satellites and field measurement programs. The EOSDIS will provide data archive, distribution, and information management services for the MTPE program. The volume of data in EOSDIS will be enormous, with more than one terabyte received, processed, and archived every day. The database is projected to grow to 10 petabytes within 15 years. In terms of both the quantity of data accumulated for study and the overall cost, MTPE is the largest scientific project in the world.

Initially, it was assumed that EOSDIS data would be accessed by a few hundred primary EOS Earth science investigators and approximately 10000 additional global change researchers to carry out basic research. Application areas of nonresearch users such as education, agribusiness, community planning, transportation, forestry, fisheries, wildlife managers, and others, were not included. Since EOSDIS data belongs to the public, anyone who wants to access it should be able to do so. Thus, the user community will potentially number in the millions [14].

The large number of users and the huge volume of data expected for this system will require networks and storage devices with very high capacity. Clearly the network and storage components of EOSDIS will dictate the kind of applications that can be supported in this environment. Another driving consideration will be the number of simultaneous users. As the number of concurrent users grow, the system must scale to handle the increased load. One can draw an analogy of Internet activity resulting from the recent event of the Shoemaker-Levy 9 Comet colliding with Jupiter. From July 16, 1994 to July 25, 1994 , over 974770 network connections were made to servers at Jet Propulsion Laboratories in Pasadena, CA, by Internet users interested in seeing pictures of this spectacular event. This kind of access only a few years ago would have been impossible (and to some unimaginable).

The purpose of this paper is to describe the design of a network architecture to satisfy the EOSDIS program requirements and to discuss some of the applications that will be developed. The remainder of this paper is organized as follows. Section Il contains an overview of the EOSDIS project and describes some of the challenges faced by the project's developers at NASA and at Hughes Applied Information Systems, the primary contractor for EOSDIS. In Section III, we discuss 
several key requirements that have influenced the design of the EOSDIS core system architecture. We introduce a new EOSDIS component, Public Access Resource Centers (PARC's), and describe their role in the context of the EOSDIS framework. Section IV discusses the difficulty of evaluating a system that will become fully operational in 6 to $15 \mathrm{y}$ (when data from the satellites will start coming in), i.e., several generations of computing technology time, and gives some guiding principles for the developers of the system. In Section V, we characterize user communities who require access to EOSDIS data and describe the core system network architecture at several levels. Section VI introduces a specific user community, agriculture users, who exemplify a class of users that could benefit from MTPE if EOSDIS includes PARC's of the type we prescribe. We discuss how this class of users might use EOSDIS data and thus require the deployment of gigabit networks to serve their needs. In Section VII, we discuss additional EOSDIS design issues, including required computer processing power and appropriate storage structures. Section VIII concludes the paper.

\section{BACKGROUND}

\section{A. Mission to Planet Earth}

Initially referred to as "Global Habitability," Mission to Planet Earth originated in the United States in the early 1980s. In 1987 NASA released a report entitled "Leadership and America's Future in Space," commonly referred to as the Ride Report after Dr. Sally Ride, the report's chief author. The Ride Report described a concept of global monitoring called "Mission to Planet Earth." The mission concept reflected the findings of an earlier NASA report published in 1986. In this report, the Earth System Sciences Committee, a large, interdisciplinary group of scientists endorsed the idea that the Earth was an integrated single system and introduced a scientific framework for the holistic study of Earth called "Earth System Science." The Earth system science framework takes traditional areas of scientific inquiry about the Earth, it's land masses, atmospheres, and water bodies, and integrates them, paying particular attention to the areas where they interface.

During the time that United States interest in using spacebased satellites for Earth monitoring was emerging, similar interest was growing in other nations around the world. Eventually they came together and began implementing Mission to Planet Earth, which is currently envisioned as a fifteenyear effort in which the United States, Canada, Japan, and Europe are participating. The 1991 launch of the Upper Atmospheric Research Satellite is typically considered as the official beginning of MTPE. There are prospects of future Russian participation in the ongoing mission.

The centerpiece of MTPE is the international Earth Observing System (EOS), a series of polar-orbiting and lowinclination satellites that will provide global observations of the land surface, biosphere, solid Earth, atmosphere, and oceans. Additional space-based observations are being, or will be, provided by data from the Total Ozone Mapping Spectrometer; TOPEX/Poseidon, a joint U.S./French sea-level, ocean topography, and ocean color mission; the Shuttle Imaging Radar-C; and, others.

Data from the EOS satellites will be made available through the EOS Data and Information System (EOSDIS). Being built in an evolutionary manner, EOSDIS will provide data sets in Distributed Active Archive Centers (DAAC's). The DAAC's will contain data relating to atmospheric effects, sea ice and polar processes, land processes, ocean circulation, the cryosphere, hydrology, and solar irradiance, among others, with different DAAC's assuming primary responsibility for data sets related to certain areas of investigation. Recognizing the critical role anthropogenic change has on the Earth System, one DAAC, at the Consortium for International Earth Science Information Network, contains socioeconomic data to address questions raised by human land use. EOSDIS will operate pursuant to the U.S. global change data policy of full and open access for all.

The United States' contributions to Mission to Planet Earth, EOS, and EOSDIS are all part of the U.S. Global Change Research Program begun by the Bush Administration in 1990 and continued by the Clinton Administration. "Global Change" is defined by the Global Change Research Act of 1990 as "changes in the global environment (including alterations in climate, land productivity, oceans or other water resources, atmospheric chemistry, and ecological systems) that may alter the capacity of the Earth to sustain life." Through the global change research program, MTPE capabilities are being complemented with sea-level and air-based observations and in situ field work. The program is intended to provide information in the long-term "to support national and international policy formulations, as well as input to evaluate the impacts and effectiveness of these decisions."

\section{B. EOSDIS Core System}

The EOSDIS Core System (ECS) provides the underlying extendible architectural foundation and the common hardware and software building blocks to be used at the DAAC's (Fig. 1).

The key features of the EOSDIS core system are to provide:

- the ability to search for data across all DAAC's through a single interface,

- a single interface for requesting data from all DAAC's, and

- the facility to process, archive, and distribute large volumes of data.

The ECS will evolve from a series of operational prototypes, beginning with a Version 0 prototype system that became operational in mid-1994. ${ }^{1}$ The working Version 0 prototype integrates existing heterogeneous Earth science data systems and serves as a functional prototype of selected key EOSDIS services. As a prototype, it does not have all the capabilities of planned later versions; however, EOSDIS Version 0 supports use by the scientific community in dayto-day research activities and will contribute to determining

\footnotetext{
'For more information on the Version 0 Information Management System see the following World Wide Web uniform resource locator: http://harp.gsfe.nasa.gov:1729/eosdis_documents/eosdis_home.html
} 


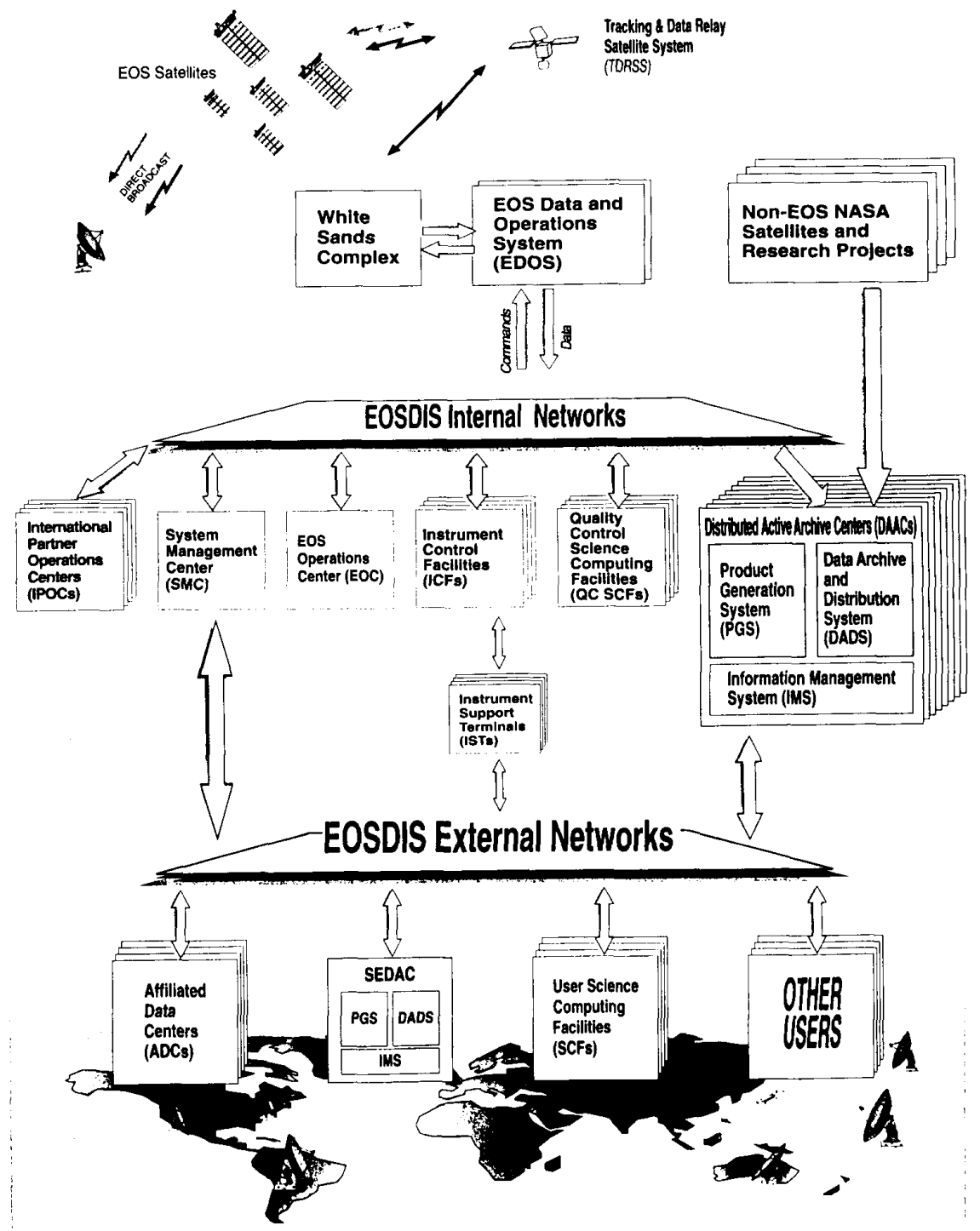

Fig. 1. EOSDIS architecture as in the 1993 EOS reference handbook.

what additional or alternative capabilities are required of future versions. EOSDIS Version 1 will be implemented incrementally beginning in 1995 . Version 1 will be designed and developed while Version 0 is operating, thus benefiting from Version 0 user feedback. It will be physically distributed but logically integrated to users. EOSDIS Version 2 will be implemented prior to the first EOS spacecraft launch in 1998. The mission and instrument command and control functions and product generation functions will be greatly enhanced and full capability will be available to support all functions of the first EOS platform.

\section{EOSDIS Challenges}

To provide uniform access to globally distributed data, the architecture of EOSDIS must take into account the wide variety of conditions under which it must operate. Reasonable response times, varying load capacities, and interoperability between diverse networking systems, data management tools, and user interface software, are project requirements. In addition, users will continuously migrate to the latest technologies, so EOSDIS must evolve to track them.

Several general assumptions can be made about the needs of EOSDIS clients and the consequent challenges faced by the project's developers.

- Interoperability is needed to ensure the accessibility of EOS data from diverse platforms. The ability of various software components (e.g., graphical display, data management, metadata browsing, statistical analysis) to communicate effectively with each other is critical to provide widespread access to this data. Although the adoption of the Hierarchical Data Format as the EOSDIS 
standard represents progress in support for heterogeneous databases, additional interoperability features are needed to provide full functionality to users on any platform [10].

- Scientific data sets are too massive to be readily accessible to most end users. For example, a typical Landsat image is about 278 Mbytes. Some industrial and scientific researchers may have systems that are capable of receiving vast data sets, but users without such large-scale systems need alternative means by which such data may be provided. The existence of on-line interactive support, with which end users can access and manipulate data without being forced to load it onto their systems, is a desirable and perhaps essential feature of the EOSDIS architecture [10].

- Transmission of extremely large data sets is slow. Although the response time often suffers wide area network delays, it can also be delayed significantly by the time required to access and transmit the dataset at the host site. Efforts must be made to minimize the time spent processing data requests at the host. Furthermore, a stronger integration of the processing power of end stations and the network communications being utilized is needed. The host's processing capabilities should not be severely taxed unnecessarily if the remote end user has a limited capacity to deal with the transmission of the results.

- Locating, recognizing and interpreting relevant information in vast data sets can be difficult. A general data browsing capability is needed to help locate appropriate data sets. In addition, an efficient browsing capability is needed so the user can cull relevant data from larger data sets [12]. An alternative method of supplying users with information regarding the existence and location of pertinent data and services is the concept of a subscription service [8], which provides automatic notification to users based upon their specified interests. An inherent advantage in the PARC concept is that users connecting to a PARC will only browse and access data pertaining to a certain area of interest. Some professionals would search for relevant data, then value-add it and provide it to end users. It is also important to provide tools that assist in the discovery of information in the archives. It is well-known that quantitative data concerning the hole in the ozone layer over the Antarctic was in place more than $10 \mathrm{y}$ before it was widely recognized, interpreted, and believed.

- Productivity is restricted by inadequate interfaces to the data. Data management environments are usually created by computer scientists, whereas analytic environments are designed by people in the particular scientific discipline that generates the data. Serious efforts to integrate the data management and analysis environments are needed.

- The EOSDIS system must be designed in such a way that upgrades using future tochnologies and methodologies are facilitated. Specific areas that EOSDIS evolution must be prepared to accommodate include: multimedia environments, heterogeneous operating systems, highperformance computing, storage, visualization, network- ing, real-time data acquisition, and advances in extended relational and object-oriented database models.

- Research methods for dealing with massive scientific data sets will differ fundamentally from traditional research methods. While the traditional approach involved designing an experiment and then generating data to be interpreted, projects such as EOSDIS involve the generation of huge amounts of data, around which researchers will then define their experiments. Rather than producing the exact set of data needed for scientific analysis, the researcher will have to devote a significant effort to the search for appropriate data among much larger databases.

- The exact location of data may not interest the user. The EOSDIS network must function such that, once a user enters it anyplace, the full data set is available to him or her with no burden to know where it resides (i.e., the data is location-transparent).

- Nontraditional users will not be familiar with advanced computing concepts. If MTPE is to benefit people outside the active research community, its information must be accessible to people for whom the computer is an aid and whose usefulness is inverse to the amount of instruction it requires.

\section{EOSDIS SYSTEM ARCHITECTURE}

Several key requirements influence the design of the EOSDIS Core System architecture. These key drivers have been developed through interactions with the science community, NASA, and Hughes Applied Information Systems; and represent factors that may determine and affect users' information needs. These drivers and constraints (resources, budgets, etc.) guide the development and evaluation of the architecture. Note that these drivers have been determined by sophisticated users. The far larger class of others who potentially could benefit, if information useful to them could be extracted with minimal knowledge of computing technologies, was not represented when specifications were derived. The drivers have been classified into the following four categories [16]:

- Science drivers: Dictated mostly by the research scientists' needs in supporting long term global change research. These are centered around the needs of research teams to effectively and efficiently find, access, use, and share the results of their research, including data, modeling results, and observations and analyses.

- Technology drivers: Based on advances in computer and communications technology that impact users abilities to search, access, and process information in a large system such as EOSDIS. These drivers also focus on evolving technologies that will affect the way the system will be used in the future.

- System engineering drivers: Determine the extent to which the system must evolve over time, and hence provide design guidelines to help determine an appropriate level of effort to devote to system expansion and flexibility. These drivers also mandate consideration of issues such as system scalability and evolvability, and of system reliability, maintainability, and availability. 
- Policy and funding drivers: Based on fundamental mission objectives and funding constraints, and include guidelines regarding expansion of the ECS system to support other uses, support of value-added providers, and commercial use of the system.

Based on the above drivers, the ECS project has developed a set of system design guidelines [16]. These guidelines may be summarized as follows. The architecture must be "seamless" and not distinguish between data, metadata (data on the data) and catalogue information. It must publish the availability of all products and allow access to them. The architecture must be open and support heterogeneous and autonomous system sub-components. System management should be decentralized (both physically, and in terms of authority). This open, distributed architecture is expected to enhance application portability and will incorporate equipment, operating systems, programming languages, applications, etc. from different vendors and developers; simultaneously it will provide users with location transparency.

Fig. 1 depicts the EOS architecture as in [9]. It should be noted that the architecture in the figure does not adequately serve the largest set of potential users of EOSDIS data. Further discussion on this and the architectural extensions we recommend are given later in this section and in the rest of the paper.

Several levels of data have been defined by the EOS Advisory Panel [9].

- Level 0-Reconstructed unprocessed instrument/payload data at full resolution with only communications artifacts removed

- Level 1-Reconstructed unprocessed instrument data at full resolution, time-referenced, and annotated with ancillary information appended to the Level 0 data

- Level 2-Derived geophysical variables at the same resolution and and location as the Level 1 source data

- Level 3-Data or geophysical variables that have been spatially and/or temporally resampled (i.e., gridded data)

- Level 4-Model output and/or variables not directly measured by the instruments but instead derived from lower level data

The raw data (referred to as Level 0 data) from instruments housed on the EOS satellites will be routed to the production facility in Fairmont, WV, where the communications headers will be stripped, the Level 0 Raw instrument data calibrated at original resolution and time ordered, and duplicate packets removed. The West Virginia facility also provides a long term backup archive for the Level 0 process data. The data then flows through the EOSDIS internal networks to several different sites with various responsibilities. The central information sites are the DAAC's and current plans call for most science users to connect to EOSDIS through the DAAC's. We propose another component to EOSDIS, Public Access Resource Centers (PARC's), to provide access to EOSDIS data to the general public.

NASA has selected eight DAAC's to process, archive, and distribute EOS and related data and to provide a full range of support to the user community. ${ }^{2}$ The DAAC's are also responsible for processing certain pre-defined data sets into products for users, and to provide permanent archives to data during the EOS operational lifetime and beyond. Each DAAC performs data processing functions, including routine generation of standard products, quick-look products, metadata, and browse data sets, in addition to its archiving and distributing responsibilities. A DAAC will offer a wide range of standard data products plus products unique to a user community for which it has special responsibility.

The Goddard Space Flight Center DAAC will hold data associated with upper atmosphere, atmospheric dynamics, global biosphere, and geophysics; the Langley Research Center DAAC will maintain data related to radiation budget, aerosols, and tropospheric chemistry; EROS Data Center will administer land processes imagery; University of Alaska-Fairbanks will hold sea ice and polar processes imagery; University of Colorado will hold all nonsynthetic aperture radar cryosphere data; Jet Propulsion Laboratory will administer ocean circulation and air-sea interactions data; Marshall Space Flight Center will hold all data relating to hydrology; and Oak Ridge National Laboratory will maintain biogeochemical dynamics data. Besides the eight DAAC's described above, an additional center, called the Socio-Economic Data and Applications Center, serves as a link between EOS and the socio-economic/policy user community.

Various research teams will analyze EOS data, obtained from DAAC's, for their own purposes, and of course, they will have some computational resources of their own. Their centers are termed Science Computing Facilities, SCF's, and will be used to develop and maintain algorithms (for both Standard and Special Products), calibrate the EOS instruments, validate data and algorithms, generate Special Products, and analyze EOS and other data in pursuit of the MTPE science objectives. The SCF's may range from single workstations to large supercomputer data centers. The ECS will provide software toolkits to the SCF's and other users to facilitate data access, transformation and visualization, and for algorithm development. Currently, NASA has adopted the Hierarchical Data Format from the National Center for Supercomputing Applications to be used in EOSDIS products. Some SCF's will play an operational role in quality control of the EOS Standard Products and these SCF's will be linked to the DAAC's via guaranteed service quality communications lines to support reliable exchange of large volumes of data.

\section{A. Public Access Resource Centers}

Notably absent in the architecture is any means or provisions by which the general public can easily access the EOSDIS data. Mission to Planet Earth appears to be a multibillion dollar project that is expected to meet the needs of $1000-10000$ scientists, but one that will benefit the populace only indirectly through these primary investigators. To facilitate access of value-added products for a wider community, we propose to

\footnotetext{
2The exact number of DAAC's depends critically upon funding. In longterm projects such as MTPE, budgets fluctuate with time. The number of DAAC's and their specific assignments are those planned as of July I, 1994.
} 


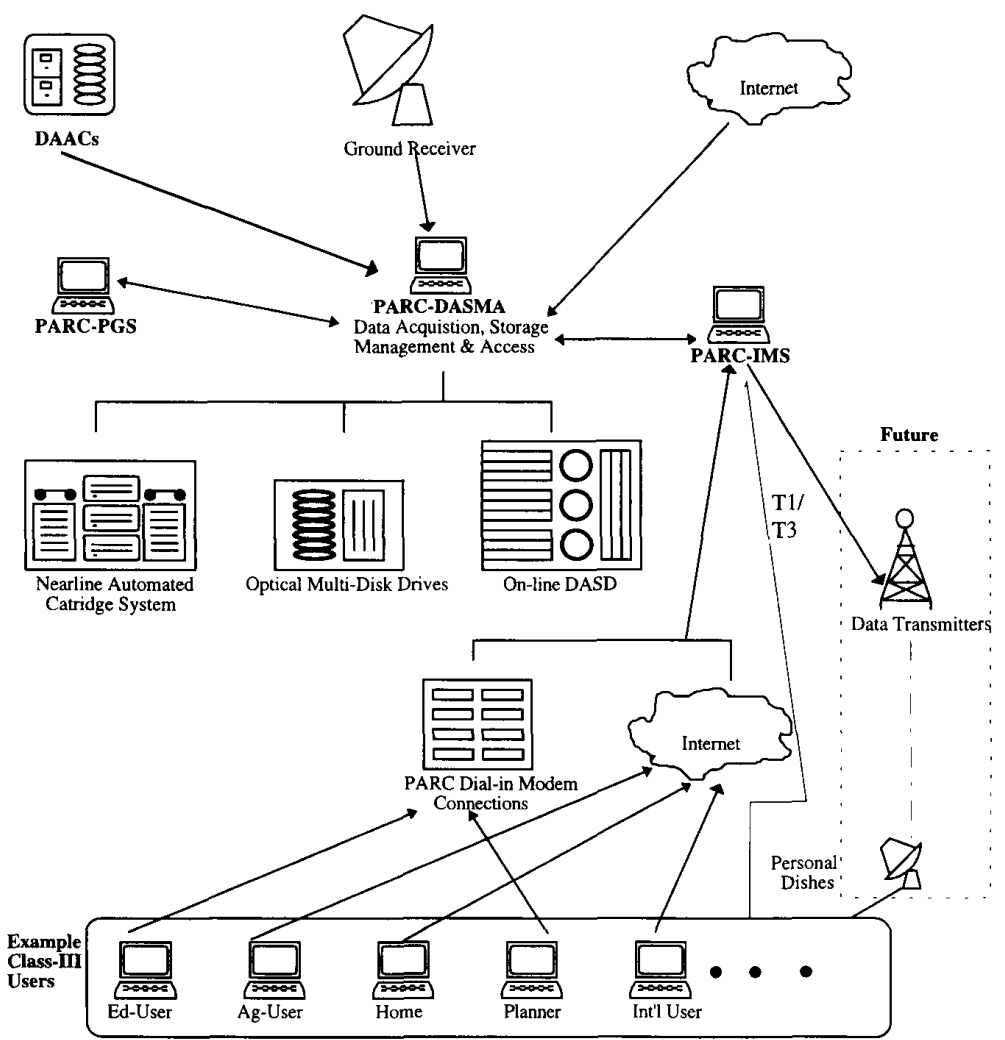

Fig. 2. Proposed architecture of a PARC.

add to EOSDIS additional data providers, called Public Access Resource Centers or PARC's.

We envision that most PARC's would have a particular application focus. For instance, an education-oriented PARC, EdPARC, would serve the K-12 and higher education community; an agriculture-oriented PARC, AgPARC, would serve agriculture, precision agriculture, and agribusiness; a planneroriented PARC, PlanPARC, would serve the government and private community planning; a home-oriented PARC, HomePARC, would serve home and household needs for weather and other EOSDIS information; and so on. Each PARC would provide value added products that meet the needs of a specific user community. The primary functions of these PARC's will be to serve the data acquisition, storage and accessibility needs of a user community and to generate exciting and useful applications for that community. Fig. 2 depicts the architecture of an AgPARC.

PARC's will differ from other data providers, e.g., the DAAC's, SCF's, etc., in the following ways. PARC's will have a much stronger active component, in which groups of scientifically capable people act as "gem miners, processors and advertisers" in the mountains of EOSDIS data. This is the principal function of the PARC-Product Generation System. PARC-Product Generation Systems mine for, invent, develop, publicize, and demonstrate data products and applications. They also educate their users in how to access the system, how to interpret the products available, and how to exchange information with others having similar interests. Not all the information will flow one way from PARC to user. In fact, an important justification for adding PARC's to the EOSDIS architecture is so that Mission to Planet Earth can be strengthened by input from creative people. The philosophy is, the more people who participate in MTPE, the greater the number of uses the information acquired will have. We will discuss user and network requirements for the PARC's later in the paper.

\section{System Evaluation}

System evaluation will be necessary on many aspects of the proposed EOSDIS system design. Certainly, the intra- and inter-DAAC communication characteristics will require some performance study and evaluation, as will the DAAC-PARC and PARC-Public communications.

The EOS sensors that will deliver large data sets are expected to become fully operational in 6 to $15 \mathrm{y}$. Over its years of operation, EOSDIS must quickly integrate new technology in storage, computing power and communication technology. Predicting the technical advances is difficult. Predicting the ways in which users will utilize the system and the data is much more difficult. Performance of specific candidate architectures can be evaluated through detailed system simulations. Performance measures typically considered include 
such things as utilization rates for communication lines and processors, queue sizes, and statistics and distributions of waiting times for satisfaction of queries and other services. We are concerned that these kinds of performance evaluation results, based on the trends and technologies of today, can easily produce architectures that are limiting and selffulfilling. The conclusions can become correct by definition, and impede the inclusion of unforeseen technology advances. Alternatively, we assert that other measures of performance, such as robustness, consistency, adaptiveness and evolvability are of high importance in EOSDIS.

We offer the following guiding principles:

1) Create widely available data access mechanisms, including a provision for domain specific application areas (such as PARC's).

2) Develop and support powerful and intuitive graphical user interfaces early on, to encourage widespread use.

3) Establish generous allocations of ports for discretionary users at all levels of the architecture, to encourage new and imaginative uses.

4) Establish standards, for such things as interfaces, protocols, and data formats, so that components can be changed or replaced as long as they meet system standards.

\section{USER AND NETWORK REQUIREMENTS}

\section{A. User Community}

There have been several attempts to characterize the future constituents and size of the EOSDIS user community [1], [13], [14]. One approach is to classify the user communities into one of the following three classes:

- Class I users, 551 principal EOS investigators, each leading a team of several coinvestigators [9];

- Class II users, the 10000 or so global change researchers not participating directly in the EOS program [9]; and

- Class III users who do not fall into Classes I or II [21].

The potential size of the EOSDIS Class III user community is in the millions. Most of the researchers (Classes I and II) will access EOS data products through the Scientific Computing Facilities (SCF's) located at the EOS investigators' home institutions. The SCF's may range from single workstations to large supercomputer data centers [15]. The SCF's will be linked to DAAC's via dedicated high speed communication lines to support reliable exchange of large volumes of data (e.g., SONET OC-3 lines). Access for Class III users will be through DAAC's, PARC's, or intermediate Internet hosts Class III users are expected to utilize the National Information Infrastructure heavily as it develops [5].

Users will search EOSDIS data using geographic coordinate references, bounding polygons, common geographic names, and data content or simply click on predefined icons as may be the case with Class III users. Searches for data coincident in time and space across data sets at the DAAC's will also be supported. In addition, users will be able to browse data sets generated by algorithms provided by EOS investigators.
TABLE I

Some EOS Satellites and Their Expected Data Rates

\begin{tabular}{lc}
\hline Satellite Series & Data Rate \\
\hline EOS-AM & $16.0 \mathrm{Mb} / \mathrm{s}$ \\
EOS-PM & $7.7 \mathrm{Mb} / \mathrm{s}$ \\
EOS-CHEM & $1.1 \mathrm{Mb} / \mathrm{s}$ \\
EOS-AERO & $26.0 \mathrm{Mb} / \mathrm{s}$ \\
EOS-ALT & $215.0 \mathrm{~kb} / \mathrm{s}$ \\
PEOS & $3.5 \mathrm{Mb} / \mathrm{s}$ \\
\hline
\end{tabular}

Although developing specific criteria for Class I and II users are important, the architecture must also simultaneously handle the requirements of Class III users. The problem of accurately estimating the future constituents and size of the EOSDIS user community is difficult. Those who have undertaken such studies have concluded that both the number of users and the number of system accesses can only be predicted roughly. Moreover, there remains disagreement over even the broad conclusions. We have little confidence in our ability, or the ability of others, to predict what the utility of EOSDIS will be for users far into the future. We believe that the emerging "webs" of information sources and services, such as the World Wide Web, will lead to altered user behaviors that no one can predict. Previous studies in our opinion have significantly underestimated the number of potential users of EOS data [1], [8]-[10], [13], [14].

The extent to which EOSDIS should serve the Class III user community is a policy issue and specific policies regarding distribution of EOS data are not yet in place. We strongly advocate that the policies facilitate the development of applications in user groups outside the core science research community for which EOSDIS was originally conceived. With appropriate applications, Class III users of EOSDIS could number in the thousands of simultaneous users and each requiring bandwidths on the order of many megabits per second. The information generated by MTPE space-based satellites, and stored as part of EOSDIS, is estimated to exceed one terabyte per day. High speed networks will be needed to transfer this large volume of information to the required DAAC's and PARC's. Table I depicts the data rates for a few of the EOS satellites that will be launched by the end of the decade [9].

\section{B. Network Architecture}

The ECS network architecture must support existing protocols and network standards whenever possible (e.g., OSI and TCP/IP) and yet be amendable to the rapid advances of computer network technology. Recent advances and standardization in broadband network technologies has laid the groundwork for a whole new generation of networks to be realized. These networks will have a significant impact on the protocols, services and bandwidth available to users of computer networks.

ECS network design goals include [16]:

- a scalable, flexible, and enduring network architecture;

- seamless LAN/WAN integration; 
- an easy, low-risk migration capability;

- high network availability with any-to-any connectivity; and

- high performance, low delay with bandwidth allocated on-demand.

The ECS network will support diverse data sets useful to a broad range of users. This combination of data and user diversity is one of the major challenges for system designers. Users will access EOSDIS data through DAAC's, on-line SCF's, or PARC's. ECS network connectivity can be viewed at three levels: 1) DAAC-with-DAAC, 2) PARC-with-DAAC, and 3) User-with-PARC. ${ }^{3}$

At the highest level, the network is viewed as the interconnection of a small number of DAAC's (the current number of NASA-approved DAAC's is eight) in the form of an irregular topology with dedicated communication links. Today all interDAAC communication links are fractional $\mathrm{T} 1$ or full $\mathrm{T} 1$ lines. To support the transmission of large data sets and enable realtime collaboration among research scientists working at the various DAAC's, upgrades will have to be made to existing communication network links. It is expected that inter-DAAC communication links will evolve to asynchronous transfer mode (ATM) technology (e.g., $155 \mathrm{Mb} / \mathrm{s}$ OC-3 or $622 \mathrm{Mb} / \mathrm{s}$ OC-12 links) and support bandwidth-on-demand in the near future [16].

At the second level, PARC's are added to the network architecture. Initially, PARC-with-DAAC connectivity will be carried out through existing Internet links. In the future, PARCwith-DAAC connections will utilize transport mechanisms developed as part of the National Information Infrastructure. These mechanisms will presumably be ATM and based on Broadband Integrated Services Digital Network (B-ISDN) standards. The volume of data traffic on PARC-with-DAAC communication links will depend, in large part, on the application areas of interest at the PARC's. For example, applications utlizing an AgPARC (see discussion in the next section) will require transmission rates exceeding $100 \mathrm{Mb} / \mathrm{s}$ and have on the order of several hundred simultaneous users [21].

At the third level, communications infrastructure for Class III users is added to the network architecture. This infrastructure will consist of a wide variety of systems. From systems of modems and copper telephone lines to hybrid systems of coax, copper and fiber to all-digital advanced communication systems based on B-ISDN standards. Though we expect Class III users will utilize PARC's for most of their communication needs, they will also be capable of connecting to a DAAC directly. Clearly, the Public-PARC interconnection network will be varied and diverse. In the near-term, many end-users will have only dial-up telephone access. This will impact the type and quality of services they receive. We anticipate user access to EOSDIS data will improve over time as technology improves and costs decline.

Consider, for example, the telecommunications infrastructure supporting individual farmers and ranchers today. These users have access to a wide variety of personal computers

\footnotetext{
${ }^{3}$ The focus of this three-level model is on enhancing the DAAC's as the archive components and PARC's has value-adders.
}

DAAC

UNI

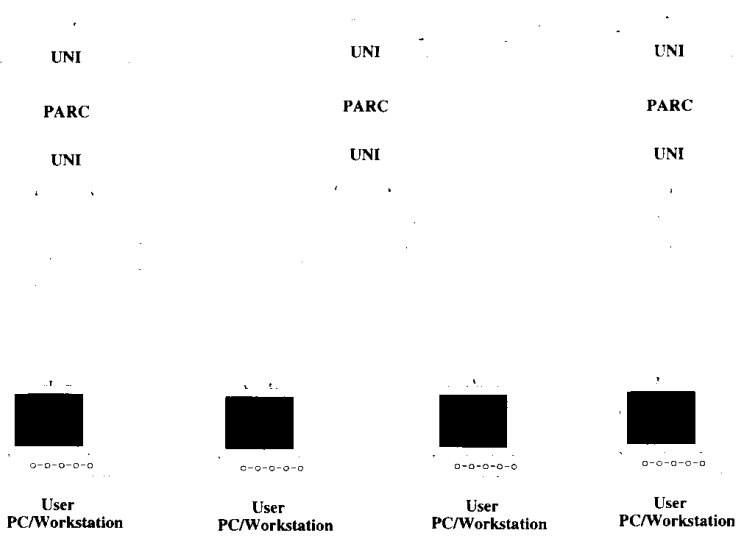

Fig. 3. User-PARC-DAAC interconnection model.

and computing equipment, but primarily one communication medium, the telephone system. Because they are geographically isolated, individual farmers and ranchers must achieve connectivity to their personal computers with the use of modems attached to their telephone lines. Today, it is possible to achieve data throughput rates as high as $38800 \mathrm{~b} / \mathrm{s}$ over regular voice grade telephone lines. It is also likely that some Class III users will have $18^{\prime \prime}$ satellite dishes to receive data. In the future, the Integrated Services Digital Network (ISDN) will become commonplace in rural communities and provide higher data rates over existing twisted pair copper cabling than is possible today over the existing analog telephone system. ISDN will enable new end-user applications to be realized (e.g., high-speed file transfer, multidocument image storage and retrieval, telecommuting, and video conferencing). With the introduction of ISDN and B-ISDN connections to the home, Class III users will have improved network capabilities allowing fast access to large image data sets and enabling remote video conferencing among their peers and associated PARC personnel.

In Fig. 3 we show, conceptually, how a DAAC and associated PARC's might be interconnected. The user-to-network interfaces (UNI's) in this model can be classified into several categories. The choice of UNI for a particular network connection will be based on the expected load, traffic type, cost, and distance to the DAAC. The UNI's can be modem banks (phone lines supporting various baud rates), interfaces based on T1 or T3 standards, multirate ISDN interfaces, or ATM interfaces. The choice of interface will be driven by the traffic requirements and the cost/benefits expected from any given link. 
The PARC's are a vital component in the telecommunications infrastructure we propose to support Class III users. DAAC's will be consumed with "push" activity, with generating a subset of standard products, and with providing some "pull" capability (i.e., data delivery) to the 551 EOS Class I scientists and perhaps to the 10000 Class II users. At best, everyone else, the Class III users, will be limited to passive use. The archiving function of DAAC's must have higher priority than any of these tasks, further limiting their ability to serve Class III users.

Without PARC's, users will have limited, passive access to the DAAC's (e.g., through a Mosaic-like interface). Although this may be sufficient for some users, we believe that this is unnecessarily restrictive for all users and will ultimately limit usage. We should not overlook, either, that MTPE will not just benefit people, but also they will benefit MTPE. The PARC's will "develop" users and provide "customer support." The overwhelming majority of people will not even know a Mission to Planet Earth exists, let alone know that they could use some of the data the mission collects. Thus, one of the important functions of a PARC will be to further educate the public about MTPE. Also, users of the PARC's, through mining the data, will discover and invent uses for EOSDIS data that are unforeseen at this time. This will also aid in furthering the goals of MTPE.

Agriculture is a specialization area that can benefit greatly from a PARC. We envision that an AgPARC would drastically improve the methods and means by which farmers plant, nurture, harvest, transport, and market their products. Farmers could assess market and yield trends more scientifically and more readily than today, which would result in improving the economic welfare of the agricultural community as well as the nation and the world. This is especially critical today, since the size of an average farm is on the increase while the number of farm workers continues to decrease. The role of EOSDIS in agriculture is discussed further in the next section.

\section{EOSDIS APPLICATIONS IN AGRICULTURE}

EOS users working in agriculture and natural resource applications will benefit enormously from remotely sensed EOSDIS data. The utility of remote sensing in agriculture and natural resource management is illustrated by the use of Landsat multispectral scanner system imagery in the U.S. to assess the Soviet Union winter wheat harvest during the early 1970's [4]. Since then, Landsat multispectral scanner data, followed by Thematic Mapper imagery, and later SPOT multispectral data have been used extensively to derive various agricultural parameters, such as crop yield, crop stress, and crop growth [3], [19]. Research by Milton et al. [18] and Shibayama and Akiyama [22] among others has shown that spectral information derived by remote sensing systems can predict crop yields and detect crop diseases with a high degree of correlation between actual and projected yields. EOS data promises to be quite valuable in crop phenology and other fundamental scientific fields related to agriculture.

Source [11] provides some indication of the wide use of geographic data for agricultural and natural resource appli- cations in Federal government agencies. There are too many uses of these data to be listed here, but citing some of the agencies illustrates the broad applicability of EOS data. Participating agencies using spatial data products directly related to agriculture and natural resources include the Agricultural Stabilization and Conservation Service, the Forest Service, the Soil Conservation Service, the Census Bureau, the Bureau of Economic Analysis, the National Weather Service, the Bureau of Land Management, the Fish and Wildlife Service, the National Park Service, and the Geological Survey. State and local governmental bodies have agencies with similar interests. For example, the Nebraska GIS Interest Group has approximately 300 members including representatives of state governmental agencies such as the Game and Parks Commission, the Natural Resources Commission, the Natural Resource Districts, the Agriculture Department, the Department of Environmental Control, the Water Resources Department, tribal councils, the Governor's Policy Research Office, and the State Legislature. to name a few. At the county and city level, potential government users of EOSDIS include extension agents, assessors, planners, engineers, and surveyors.

Note that the exploiters of these rich information resources are, to date, agencies or organizations. The National Information Infrastructure offers the possibility for changing this. In the future, individuals will be able to receive the information on which they can base critical decisions about their own crops. When they lose some of their dependence on larger entities-and PARC's could help them do so--rural economic development could head in a new direction. The significance of AgPARC's to the quality of life in rural areas could be profound.

There are many potential users of EOS data in commercial enterprises involving agriculture and natural resources. Currently, a major source of geographic information for these organizations are the Regional Climate Centers funded by the National Oceanic and Atmospheric Administration and other climate and weather centers across the country. The High Plains Climate Center at the University of Nebraska-Lincoln, the Regional Weather Information Center at the University of North Dakota, and other similar centers provide data products such as temperature and precipitation statistics and probabilities, temperature thresholds, growing degree days, insect degree days, soil moisture, evapotranspiration, water supply outlooks, crop projections, fire danger, and so on. Some of the uses are obvious, for example, a large purchaser of grains makes national production estimates. Other applications are less obvious. For example, a corn-packing plant uses climate and crop information to estimate dates of maturity, so that it can optimally schedule transportation at harvesttime.

These existing climate centers point the direction for public use of EOS data, not only in agriculture and natural resource management, but for other applications including education. A central characteristic of these centers is that they integrate data from a variety of sources-remote sensing data, soil type maps, elevation maps, weather reports, historical climate and weather information, in situ measurements, historical crop data, etc.- to generate products that are of great practical 
utility and they make the products accessible to people with limited knowledge of computer systems.

As a specific example of the utility of EOS data, consider the problem of estimating soil moisture. Crops require variable quantities of water at different stages in the growth cycle and depending on climate. Soil moisture is a critical consideration in planting strategies, crop selection, fertilizer application, and irrigation. For quantifying potential economic benefits, McQuillan suggested that accurate predictions of drought could be worth as much as $\$ 3000000000$ per year in agriculture [7] and soil moisture has other applications, for example in hydrology to assess flood danger. Soil moisture can be measured in situ and with remote sensing, especially with radar-equipped satellites such as the Earth Resources Technology Satellite-1 [7]. In situ measurement techniques are well-developed, but dense sampling of large areas is impractical. Remote sensing data can provide increased spatial coverage and resolution. When the soil moisture is integrated with soil type, climate conditions, high-resolution rainfall estimates (e.g., using ground radar stations), and crop growth data (e.g., current crop-specific rooting depth) a model of crop phenology can be constructed for individual farmers. Other EOS data, for example moderate resolution imaging spectroradiometer and advanced spaceborne thermal emission and reflection radiometer, will be useful both in developing and using such models for assessing crop growth and conditions. The same data and models might be used on a larger scale by water resource managers to predict and/or control water utilization.

Acquiring vital information without the need for intervening organizations is the promise of the National Information Infrastructure. In the case of agriculture, it could put individual farmers on an equal status with large farming conglomerates.

\section{TECHNICAL DISCUSSION}

The expectations of end-users in specific application domains, such as agriculture, will be high. To be successful, a PARC must offer users a consistent computing environment that will transparently access data on their local machine and on widely distributed hosts. The data must be supported by tools that provide analysis, decision support and visualization all within the context of their application. Furthermore, the architecture must accommodate widely distributed heterogeneous platforms with heterogeneous databases. The system design is complicated by needs for high-performance computing, massive storage, and high speed communications, making it essential that significant advances be achieved in these areas and be employed as quickly as possible. In addition, software technology for management of storage and database manipulation must also make dramatic improvements. Development of and adherence to standards is critical to achieving acceptable performance of the system. In this section we describe these issues, and suggest the ways in which they influence the design.

Substantial processing power will be needed at DAAC's and PARC's that choose to process and store complete sets of data for any of the data-intensive instruments. For example, the
Enhanced Thematic Mapper Plus instruments are expected to capture 300 images each day. These images will involve about 837 Gbytes of raw data, and require processing for radiometric, geometric, atmospheric and terrain corrections. Relative to present capabilities, the computational ability (processing power and numerical methods) to carry out these corrections must improve greatly just to keep up with the flow of data from the satellites. Applications that require pixel-level processing of such sets of images will need similar processing power. For example, many applications require analysis involving proximity-determining such things as the likelihood that specific attributes are spatially autocorrelated. Using color or reflectance to monitor crop progress or model the onset of a disease would involve such analyses. Computation of the similarity measures for the analysis requires pixel-level processing, either to directly compute the measure or to vectorize the image in preparation for the computation [6].

In terms of standards, it is desirable that all affiliated facilities adopt the same data structure and sequencing convention for image storage. There are a host of competing data structures, such as quad-trees, $k$ - $d$ trees, KDB-trees, $R$ trees, grid files, and RMD-trees. ${ }^{4}$ Such data structures utilize a sequencing convention such as Morton coding to avoid storing tree pointers and keys. Convention differences as simple as one site ordering a quadtree sequence beginning with the Northwest quadrant and another with the Southeast quadrant or choosing "left" versus "right" sequencing would force significant data conversion processing, even though the representations are equivalent.

It is likely that multiple computers, each employing commodity RISC processors in parallel, will be the computers of choice for the compute-intensive functions, considerably different from many supercomputers today. Compute cycles are not likely to be particularly expensive. Rather, development of parallel software and systems management to effectively employ such systems will be the primary challenge.

In sheer size, the EOSDIS far exceeds the scale of any database system previously designed or operated. Fortunately, densities of storage devices are likely to dramatically improve before EOSDIS is fully on-line, making it possible to archive, access and maintain these large data sets. In particular, giant magnetoresistance will likely improve magnetic disk capacity by about a factor of 17; and other improvements in capacity and cost are expected to maintain a rate of approximately $60 \%$ each year. Thus, large portions of the EOSDIS data could actually be maintained on magnetic disk. Capacity and access times for magnetic tape, optical disk, optical tape, and CD-ROMS are also expected to significantly improve by the time EOSDIS is on-line. Juke boxes and robotic devices for physically handling large numbers of disks and tapes for near-line access are steadily improving and are essential in the design. Transfer rates will almost certainly be more of a bottleneck than capacity.

Software issues in both database management and systems storage administration will likely be more critical than storage capacity per se. Searches based on location and instrument

\footnotetext{
${ }^{4}$ Trade-offs and further references are available in [20].
} 
type are predictable, and efficient indexing must be supported for such searches. Searches based on content are very difficult to predict, making it certain that sequential searches through enormous data sets will regularly be necessary. Intelligent systems that can optimize the effectiveness of each sequential search by assembling responses to multiple queries can be of great benefit. Software to guard against runaway queries that bog down the system must be very intelligent, and represent a basic software challenge in the EOSDIS design. Data compression and browse capabilities are closely related. Fast image previewing at low resolution is a highly desirable feature to offer the user. It may be desirable to utilize lossy compression techniques to actually pre-compute and archive highly compressed versions of many of the more popular images, to make them available for viewing interactively over the network without incurring the real-time processing overhead of a compression algorithm. In addition to metadata, users should be supplied with complete subsetting environments that enable them to understand the background computational effort involved in supporting their queries, thereby promoting efficient and effective use of the system.

Use of redundant data across media will likely be of great benefit, especially at the domain-specific PARC's. Given the vastness of the data maintained by EOSDIS, replicating data may seem an absurdity. However, as with virtually all large database systems, most of the I/O will almost certainly be for a relatively small percentage of the database [2]. Some data, such as files that catalog user activity or provide metadata, will likely be accessed by nearly all users and must be on disk. Overall system performance is limited by the maximum rate of the busiest medium. Thus, replication of such data is essential, lest many volumes function at rates far below their capabilities. Data striping and various redundant arrays of inexpensive disks (RAID) techniques will be important in effectively using the storage devices within the system, even across the widely distributed system. The desirability of this is highly dependent on the ratio of reads to writes into storage, because in a replicated set a read needs to access only one file, while a write must access them all. Again, information such as metadata, index files and catalog data will have high read/write ratios, suggesting that substantial replication will be an advantage. The opposite will likely be the case for much of the image data itself, although some high-level valued-added image products at the PARC's will be exceptions. Hierarchical storage management (HSM) will be beneficial only if there will be large differences in access times between tertiary and secondary storage, which is doubtful. EOSDIS, including the PARC's, should plan to fully support complete on-line data concerning major projects and events while they are of high interest. This may be handled more through attentive system administration than through HSM techniques. Data warehousing refers to automatic maintenance of a specialized value-added database from a larger more detailed database, especially for decision support purposes [17]. In the case of PARC's, a warehouse would likely contain relational data as well as image data, drawn from a variety of sources. Within PARC's, data warehousing is an appealing approach to consistently supporting baseline capabilities for the application area without incurring major support effort. Well-designed data warehouses, in conjunction with regular feedback from users, could be the difference between success and failure for a PARC.

Finally, the importance of standards must again be emphasized. Given the highly decentralized, heterogeneous nature of the EOSDIS effort, the operational system could easily be tremendously burdened by unnecessary overhead associated with such things as the conversions associated with supporting gateways between differing database management system engines, alternative formats for storing compressed images, and differing network transmission formats. Interoperability is a largely unresolved major issue in distributed computing. EOSDIS may be the largest interoperability challenge in history.

\section{CONCLUSION}

Many applications will be developed as part of the Mission to Planet Earth Project. Data collected and maintained by the EOSDIS will be accessed by several hundred EOS Earth science investigators, approximately 10000 global change researchers, and potentially millions of other users outside the traditional research community. It is important that the overall system architecture support the large number of users and huge volume of data expected to be collected and maintained by this system. The network and storage components of EOSDIS will dictate the kind of applications that can be supported in this environment.

Agriculture users exemplify a class of users that could benefit immensely from MTPE if EOSDIS allows easy access. We believe general access to EOSDIS data can be simplified by the existence of PARC's and the proposed National Information Infrastructure. This network infrastructure will open up vast new markets and opportunities for information services to many strategic areas, including agriculture.

\section{ACKNOWLEDGMENT}

The authors wish to recognize G. Bell, M. Hennebry, S. Henninger, L. Osborne, P. Revesz, P. Romig, J. SalasManzano, A. Samal, and S. Thompson for their participation and contribution to this work.

\section{REFERENCES}

[1] B. Barkstrom, "A preliminary EOSDIS user model," NASA Langley Research Center, 1991.

[2] K. Bates, "RAID: The new balance of power," DEC Professional, vol. 13, no. 1, 1994.

[3] E. J. Brach, "Measurement of agricultural crops by remote spectral techniques," Remote Sensing Earth Resources, vol. 3, pp. 319-337, 1974.

[4] J. Campbell, "Introduction to remote sensing" New York: Guilford, 1987.

[5] C. Christian and S. Murray, "The earth data system and the national information infrastructure testbed," Inform. Syst. Newslett., Feb. 1994.

[6] Y. Ding and A. Stewart Fotheringham, "The integration of spatial analysis and GIS," Computing Environments, Urban Syst., no. 16, 1992.

[7] F. J. Eley, R. Granger, and L. Martin, "Soil moisture: Modeling and monitoring for regional planning," National Hydrology Research Centre, Saskatoon, Saskatchewan, 1992

[8] M. Elkington, R. Meyer, and G. McConaughy, "Defining the architectural development of EOSDIS to facilitate extension to a wider data 
information system," Hughes Applied Information Systems, EOSDIS Core Syst. Project Tech. Paper 194-00131, Apr. 1994.

[9] EOS Ref. Handbook, National Aeronautics and Space Administration, Earth Science Support Office, Washington, DC, Mar. 1993.

[10] ESDIS Project, EOSDIS Version O (V0): Lessons Learned-Vers. 1.0, NASA Goddard Space Flight Center, Apr. 1993.

[11] Federal Geographic Data Committee, "Manual of federal geographic data products." Falls Church, VA: Vigyan, 1992

[12] J. C. French, A. K. Jones, and J. L. Pfaltz, "Scientific database management (panel reports and supporting material)," Univ. Virginia, Charlottesville, Dept. Comput. Sci., Tech. Rep. 90-22, Aug. 1990.

[13] Hughes ECS Team, "ECS user/data model: Approach and plan white paper," Hughes Appl. Inform. Syst., Inc., Nov. 12, 1993.

[14] P. G. Thome and T. Wingo, "Projected use of EOSDIS by U.S. NonScience Communities," Hughes Appl. Inform. Syst., Inc., Apr. 4, 1994.

[15] Hughes ECS Team, "ECS Science requirements summary," Working White Paper, FB9402V2, Hughes Appl. Inform. Syst., Inc., Feb. 1994.

[16] Hughes ECS Team, "EOSDIS core system communications and system management architecture," Hughes Appl. Inform. Syst., Inc., Mar. 1994.

[17] W. Inmon, "Building the data warehouse," QED Tech. Publ. Group, 1992

[18] N. M. Milton, B. A. Eiswerth, and C. M. Ager, "Effects of phosphorous deficiency on spectral reflectance and morphology of soybean plants," Remote Sensing Environment, vol. 36, no. 2, pp. 121-127, 1991.

[19] G. E. Moreton and J. A. Richards, "Irrigated crop inventory by classification of satellite image data," Photogrammetric Eng., Remote Sensing, vol. 50 , no. 6, pp. 729-737, 1984.

[20] Y. Nakamura, S. Abe, Y. Ohsava, and M. Sakauchi, "Efficient hierarchical structures for spatial objects: MD-tree and RMD-tree," Univ. Tokyo, Tokyo, Japan, 1993

[21] G. Seielstad et al., "Independent architecture study of EOSDIS final report: A national PARC systems for empowering the general public to use EOSDIS," Earth System Sci. Inst., Center Aerospace Studies, Univ. North Dakota, Grand Forks, Aug. 1994.

[22] M. Shibayama and T. Akiyama, "Estimating grain yield of maturing rice canopies using high spectral resolution reflectance measurement," Remote Sensing of Environment, vol. 36, no. 1, pp. 45-53, 1991.

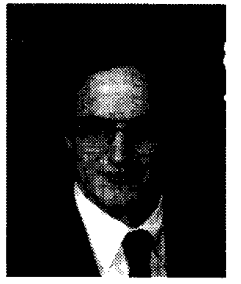

R. Vetter received the B.S. and M.S. degrees in computer science from North Dakota State University (NDSU), Fargo, in 1984 and 1986, respectively, and the Ph.D. degree in computer science from the University of Minnesota, Minneapolis, in 1992.

$\mathrm{He}$ is an Assistant Professor in the Department of Computer Science and Operations Research, NDSU. His research interests include high-performance computing and communications, multimedia systems, and high-speed optical networks. He has published more than 25 journal, conference, and

technical papers.

Dr. Vetter served as Guest Editor for the Feb. 1995 issue of Communications of the ACM on ATM networks and is an Associate Technical Editor for IEEE Communications Magazine. He is on the Board of the IEEE Computer. He is a member of the ACM and the IEEE Computer and Communication Societies.

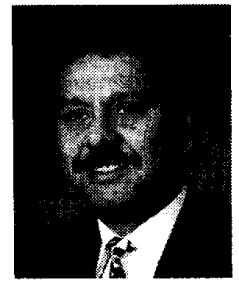

M. Ali received the B.S. degree from Leicester Polytechnic (U.K.) and the Ph.D. degree from Nottingham University (U.K.) in 1975 and 1982, respectively, both in computer science

$\mathrm{He}$ is an Associate Professor and Chairman of the Department of Computer Science at the University of North Dakota, Grand Forks. His research interests include distributed and parallel computing and computer performance evaluation and simulation. He has publications in the areas of system performance and simulations. He is coauthor of a recently published book on the Xwindow system called "Xlib by Example" (New York: Academic).

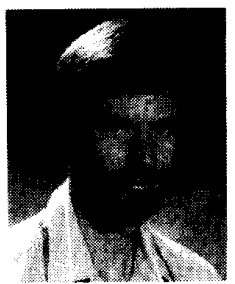

M. Daily received the B.S. degree from the University of Idaho, Moskow, and the M.S. degree from the University of New Mexico, Albuquerque, in 1983 and 1985, respectively, both in computer science.

$\mathrm{He}$ is a Research Project Manager in the Information Sciences Laboratory of Hughes Research Laboratories, Malibu, CA. He has published more than 20 technical papers on the topics of computer vision and virtual environments. His research interests include advanced human-system interfaces, virtual environments, and computer perception.

Mr. Daily has been a member of the Program Committee for severa conferences and workshops, including the 1993 IEEE Virtual Reality Annual International Symposium. He is a member of the IEEE Computer Society and Sigma Xi.

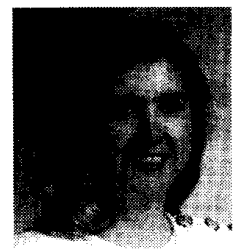

J. Gabrynowicz received the Juris Doctor degree from the Benjamin N. Cardozo School of Law of Yeshiva University, New York, NY, and the B.A degree in history and literature from Hunter College, New York, NY.

She is an Associate Professor in the Department of Space Studies at the University of North Dakota, Grand Forks. She speaks regularly on remote sensing law and policy and has published a number of papers on the subject. As a NASA/ASEE faculty fellow at Goddard Space Flight Center, Prof Gabrynowicz is researching the remote sensing laws of the major remote sensing nations.

Dr. Gabrynowicz was a member of the Congress of the United States Office of Technology Assessment Earth Observations Advisory Panel. She is currently a member of the National Research Council panel studying transborder data policy.

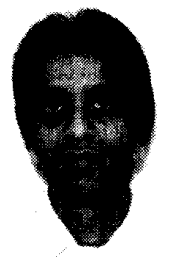

S. Narumalani received the M.A. degree from the University of Georgia, Athens, and the Ph.D. degee from the University of South Carolina, Columbia.

$\mathrm{He}$ is an Assistant Professor in the Department of Geography, University of Nebraska, Lincoln. His research interests are the use of remote sensing and GIS techniques for environmental applications. A present he is involved in several projects that focus on evaluating biodiversity, ecological mapping, ecosystem-based assistance, and wetland resources management across the United States.

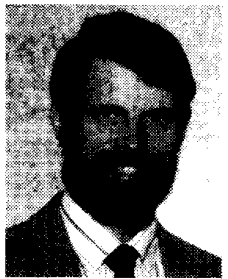

K. Nygard received the B.S. degree in mathematics and physics from Moorhead State University, Moorhead, MN, the M.A. degree in mathematics from Mankato State University, Mankato, MN, and the $\mathrm{Ph} . \mathrm{D}$. degree in operations research from Virginia Polytechnic Institute and State University, Blacksburg.

He is the Director of the Scientific Computing Center (SCC) at the Center for Aerospace Sciences, University of North Dakota, Grand Forks, and is also Professor of Computer Science. He is on leave from the Department of Computer Science and Operations Research, North Dakota State University, Fargo. He is widely published, and has served as principal investigator for more than 25 grants and contracts from the NSF, Air Force, Navy, ARPA, DOT, and other agencies. His research interest are in high-performance computing, network optimization, heuristics, artificial intelligence, and routing and scheduling applications.

Dr. Nygard is an Advisory Editor for the Journal on Heuristics and serves on the Editorial Board for the Kluwer Publishing Company's book series on computers and operations research. He is currently serving on the National Council of the Computer Science Technical Section of INFORMS (Institute for Operations Research and Management Science). He is a member of ACM IEEE Computer Society, and INFORMS 


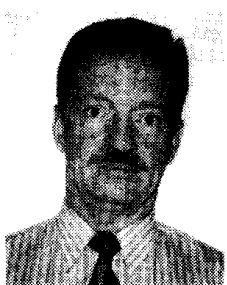

W. Perrizo received the B.A. degree from St. John's University, Collegeville, MN in 1965, the M.S. degree from the University of Wisconsin, Madison, in 1967 , and the Ph.D. degree from the University of Minnesota, Minneapolis, in 1972.

$\mathrm{He}$ is a Professor of Computer Science at North Dakota State University, Fargo. Perrizo has published more than 100 journal and conference papers. His research interests include database systems, distributed database systems, multidatabases, multimedia, and networks.

He is a member of ACM, IEEE Computer Society, ISCA, and ISMM.

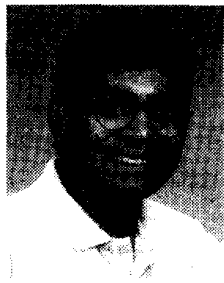

P. Ram (S'93-M'93) received the B.E. (Hons) degree from the Regional Engineering College, Tiruchirapalli, India, in 1988, and the M.S. and Ph.D. degrees from North Dakota State University, Fargo, in 1991 and 1993, respectively, all in computer science.

He is currently an Assistant Professor in the Department of Computer Science, University of North Dakota, Grand Forks. His research interests include database query optimization, multimedia and hypermedia systems, and transaction-management issues, particularly in multidatabase and parallel environments. He has published several articles in ACM, IEEE, and other organization-sponsored journals and conferences.

Dr. Ram is a member of the IEEE Computer Society, ACM SIGMOD, ACM SIGCSE, and UPE Honor Society.

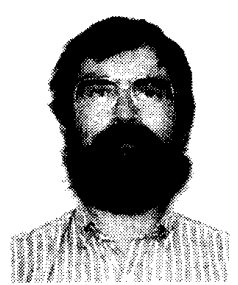

S. Reichenbach (S'85-M'89) received the B.S. de gree from the University of Nebraska. Lincoln, the M.S. degree in computer science from Washington University, St. Louis, MO, and the Ph.D. degree in computer science from the College of William and Mary, Williamsburg, VA (1989)

$\mathrm{He}$ is an Assistant Professor with the Computer Science and Engineering Department. University of Nebraska, Lincoln. His current research interests are automated image restoration, compression, and analysis of remotely-sensed multispectral data and thermal images.

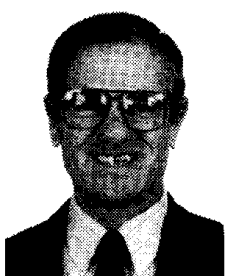

G. A. Seielstad received the A.B. degree from Dartmouth College, Hanover, $\mathrm{NH}$, in 1959, and the $\mathrm{Ph} . \mathrm{D}$. degree from the California Institute of Technology, Pasadena, in 1963

He is Associate Dean of the Center for Aerospace Sciences, University of North Dakota, Grand Forks. From 1984 to 1993, he was Assistant Director of the National Radio Astronomy Observatory, in charge of its Green Bank, WV, facility. For 20 years prior to 1984 , he was a radio astronomer at the Owens Valley Radio Observatory of the California Institute of Technology. His research interests are astronomy, radio telescopes, interferometry, and earth system science. He is the author of two books and coeditor of a third. He has published approximately 70 articles in professional journals.

Dr. Seielstad is a member of the American Association for the Advancemen of Science, the American Astronomical Society, the International Astronomical Union, and the International Union of Radio Science.

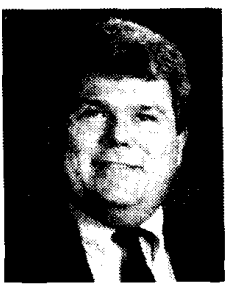

W. White (M'91) received the B.A. degree in mathematics from the University of Louisville Louisville, KY, in 1979, the M.S. degree in mathematics and the M.S. and Ph.D. degrees in computer and information science from the Ohio State University, Columbus, in 1983, 1985, and 1989, respectively.

After completing his doctoral research, he worked for three years in the field of network performance analysis at IBM in Research Triangle Park, NC. H is an Assistant Professor at the University of North Dakota, Grand Forks, and has research interests in parallel rendering, scientific visualization, and data communications and networks. He is a member of the $\mathrm{ACM}$ and the IEEE Computer and Communications Societies. 